\title{
Global behavior for a strongly coupled model of plankton allelopathy
}

Yujuan Jiao

Correspondence: jsjyj@xbmu.edu.cn College of Mathematics and Computer Science, Northwest University for Nationalities, Lanzhou 730124, People's Republic of China

\section{Abstract}

In this article, we consider a strongly coupled model of plankton allelopathy. Using the energy estimates and Gagliardo-Nirenberg-type inequalities, the existence and uniform boundedness of global solutions for the model are proved. Meanwhile, the sufficient conditions for global asymptotic stability of the positive equilibrium for this model are given by constructing a Lyapunov function.

AMS Subject Classification (2000): 35K57; 35B35; 92D25.

Keywords: plankton, allelopathy, cross-diffusion, global solution, uniform boundedness, stability

\section{Introduction}

The effects of toxic substances on ecological communities is an important problem from an environmental point of view. In 1996, Chattopadhyay [1] modified two species Lotka-Volterra competitive system by considering that each species produces a substance toxic to the other but only when the other is present and the modified model takes the following form

$$
\left\{\begin{array}{l}
\frac{d u}{d t}=u\left(a_{1}-b_{11} u-b_{12} v-e_{1} u v\right) \\
\frac{d v}{d t}=v\left(a_{2}-b_{21} u-b_{22} v-e_{2} u v\right)
\end{array}\right.
$$

where $a_{i}, b_{i j}$, and $e_{i}(i, j=1,2)$ are positive constants, $u(t), v(t)$ denote the population density of two competing species; $a_{1}, a_{2}$ are the intrinsic growth rates of two competing species; $b_{11}, b_{22}$ are the rates of intra-specific competition of the first and the second species, respectively; $b_{12}, b_{21}$ are the rates of inter-specific competition of the first and the second species, respectively; $e_{1}$ and $e_{2}$ are, respectively, the rates of toxic inhibition of the first species by the second and vice versa. For more details on the backgrounds about this system, see [1].

The system (1.1) has a positive equilibrium $E^{*}=\left(u^{*}, v^{*}\right)$ if and only if

$$
\frac{b_{12}}{b_{22}}<\frac{a_{1}}{a_{2}}<\frac{b_{11}}{b_{21}}, \frac{b_{12}}{b_{22}}<\frac{e_{1}}{e_{2}}<\frac{b_{11}}{b_{21}},
$$

where

$$
u^{*}=\left(-q_{12}-\sqrt{q_{12}^{2}-4 p_{12} r_{12}}\right) /\left(2 p_{12}\right), \quad v^{*}=\left(-q_{21}-\sqrt{q_{21}^{2}-4 p_{21} r_{21}}\right) /\left(2 p_{21}\right),
$$

(C) 2012 Jiao; licensee Springer. This is an Open Access article distributed under the terms of the Creative Commons Attribution License (http://creativecommons.org/licenses/by/2.0), which permits unrestricted use, distribution, and reproduction in any medium, provided the original work is properly cited. 
and

$$
p_{i j}=b_{i j} e_{i}-b_{i i} e_{j}, q_{i j}=a_{i} e_{j}-a_{j} e_{i}-b_{i i} b_{j j}+b_{i j} b_{j i}, r_{i j}=a_{i} b_{j j}-a_{j} b_{i j}, i, j=1,2 .
$$

Chattopadhyay [1] proved that the equilibrium $\left(u^{*}, v^{*}\right)$ is globally asymptotically stable if

$$
4\left(b_{11}+e_{1} v\right)\left(b_{22}+e_{2} u\right) \geq\left(b_{12}+b_{21}+e_{1} u^{*}+e_{2} v^{*}\right)^{2} .
$$

The corresponding weakly coupled reaction-diffusion system for (1.1) is as follows

$$
\begin{cases}u_{t}=d_{1} \Delta u+u\left(a_{1}-b_{11} u-b_{12} v-e_{1} u v\right), & x \in \Omega, t>0, \\ v_{t}=d_{2} \Delta v+v\left(a_{2}-b_{21} u-b_{22} v-e_{2} u v\right), & x \in \Omega, t>0, \\ \partial_{n} u=\partial_{n} v=0, & x \in \partial \Omega, t>0, \\ u(x, 0)=u_{0}(x), v(x, 0)=v_{0}(x), & x \in \Omega,\end{cases}
$$

where $\Omega \subset \mathbb{R}^{N}$ is bounded smooth domain, $n$ is the outward unit normal vector of the boundary $\partial \Omega, \partial_{n}=\partial / \partial n$. The, constants $d_{1}$ and $d_{2}$, called diffusion coefficients, are positive, and $u_{0}(x)$ and $v_{0}(x)$ are non-negative functions which are not identically zero.

It is obvious that $\left(u^{*}, v^{*}\right)$ is the unique positive equilibrium of the system (1.4) if (1.2) holds. Tian et al. [2] proved that the equilibrium $\left(u^{*}, v^{*}\right)$ of the system (1.4) is locally asymptotically stable if (1.2) holds.

In recent years, the SKT-type cross-diffusion systems have attracted the attention of a great number of investigators and have been successfully developed on the theoretical backgrounds. The above work mainly concentrate on (1) The instability and stability induced by cross-diffusion, and the existence of non-constant positive steady-state solutions [3-5]; (2) the global existence of strong solutions [6-13]; (3) the global existence of weak solutions based on semi-discretization or finite element approximation [14-17]; and (4) the dynamical behaviors [9,10], etc.

Tian et al. [2] considered the following SKT-type cross-diffusion system

$$
\begin{cases}u_{t}=d_{1} \Delta u+u\left(a_{1}-b_{11} u-b_{12} v-e_{1} u v\right), & x \in \Omega, t>0 \\ v_{t}=d_{2} \Delta\left(v+d_{3} u v\right)+v\left(a_{2}-b_{21} u-b_{22} v-e_{2} u v\right), & x \in \Omega, t>0 \\ \partial_{n} u=\partial_{n} v=0, & x \in \partial \Omega, t>0 \\ u(x, 0)=u_{0}(x), v(x, 0)=v_{0}(x), & x \in \Omega,\end{cases}
$$

and they proved that:

(1) If $\mu_{2}<\tilde{\mu}$, then there exists a positive constant $d_{2}^{*}$ such that the equilibrium $\left(u^{*}, v^{*}\right)$ of the system (1.5) is unstable provided that $d_{2} \geq d_{2}^{*}$, the (1.2) and the following condition

$$
\left(1+d_{3} u^{*}\right)\left(b_{11}+e_{1} v^{*}\right)<d_{3} v^{*}\left(b_{12}+e_{1} u^{*}\right)
$$

hold, where $0=\mu_{0}<\mu_{1}<\mu_{2}<\cdots$ are the eigenvalues of the operator $-\Delta$ on $\Omega$ with the homogeneous Neumann boundary condition, $\tilde{\mu}=v^{*}\left(b_{12}+e_{1} u^{*}\right) / d_{1}$;

(2) the steady-state system of the system (1.4) has non-constant positive solution, if one of the following conditions is satisfied:

(i) $d_{1} \geq D_{1}$ for some positive $D_{1}\left(d_{2}\right)$;

(ii) $d_{2} \geq D_{2}$ for some positive $D_{2}\left(d_{1}\right)$; and 
(3) if $\tilde{\mu} \in\left(\mu_{n}, \mu_{n+1}\right)$ for some $n \geq 1$, and the sum $\sigma_{n}=\sum_{i=2}^{n} \operatorname{dim} E\left(\mu_{i}\right)$ is odd, then there exists a positive number $d_{2}^{*}$ such that the system (1.5) has at least one inhomogeneous positive steady-state solution if $d_{2} \geq d_{2}^{*},(1.2)$ and (1.6) hold, where $d_{1}$ and $d_{3}$ are fixed.

We are concerned with the following plankton allelopathy model with full cross-diffusion

$$
\begin{cases}u_{t}=\Delta\left(d_{1} u+\alpha_{11} u^{2}+\alpha_{12} u v\right)+u\left(a_{1}-b_{11} u-b_{12} v-e_{1} u v\right), & x \in \Omega, t>0 \\ v_{t}=\Delta\left(d_{2} v+\alpha_{21} u v+\alpha_{22} v^{2}\right)+v\left(a_{2}-b_{21} u-b_{22} v-e_{2} u v\right), & x \in \Omega, t>0 \\ \partial_{n} u=\partial_{n} v=0, & x \in \partial \Omega, t>0 \\ u(x, 0)=u_{0}(x), v(x, 0)=v_{0}(x), & x \in \Omega,\end{cases}
$$

where $\Omega$ is a bounded domain $\mathbb{R}^{N}$ with smooth boundary $\partial \Omega, n$ is the outward unit normal vector of the boundary $\partial \Omega . d_{i}, \alpha_{i j}, a_{i}, b_{i j}, e_{i}(i, j=1,2)$ are positive constants and the initial data $u_{0}$ and $v_{0}$ are continuous non-negative functions which are not identically zero. The homogeneous Neumann boundary condition indicates that the system is self-contained with zero population flux across the boundary. The parameters $d_{1}, d_{2}$ are the diffusion rates, $\alpha_{i i}(i=1,2)$ are referred as self-diffusion pressures, and $\alpha_{i j}$ $(i, j=1,2, i \neq j)$ are cross-diffusion pressures. For more details on the backgrounds about self-diffusion and cross-diffusion, one can see [8].

The local existence of solutions for the system (1.7) is an immediate consequence of a series of important articles by Amann [18-20]. Roughly speaking, if $u_{0}(x)$ and $v_{0}(x)$ in $W_{p}^{1}(\Omega)$ with $p>n$, then (1.7) has a unique non-negative solution $u, v \in C([0, T)$, $\left.W_{P}^{1}(\Omega)\right) \cap C^{\infty}\left((0, T), C^{\infty}(\Omega)\right)$, where $T \in(0, \infty]$ is the maximal existence time for the solution. If the solution $(u, v)$ satisfies the estimate

$$
\sup \left\{\|u(\cdot, t)\|_{W_{p}^{1}(\Omega)},\|v(\cdot, t)\|_{W_{p}^{1}(\Omega)}: 0<t<T\right\}<\infty
$$

then $T=+\infty$. Moreover, if $u_{0}(x), v_{0}(x) \in W_{P}^{2}(\Omega)$, then $u, v \in C\left([0, \infty), W_{P}^{2}(\Omega)\right)$.

For the following SKT system

$$
\begin{cases}u_{t}=d_{1} \Delta[(1+\alpha v+\gamma u) u]+a u(1-u-c v), & x \in \Omega, t>0 \\ v_{t}=d_{2} \Delta[(1+\delta v) v]+b v(1-d u-v), & x \in \Omega, t>0, \\ \partial_{n} u=\partial_{n} v=0, & x \in \partial \Omega, t>0 \\ u(x, 0)=u_{0}(x), v(x, 0)=v_{0}(x), & x \in \Omega\end{cases}
$$

Yamada [13] proposed four open problems:

(1) The global existence of solutions of (P) in the case $\delta>0$ and the space dimension $N \geq 6$;

(2) the global existence in the case $\gamma=0$;

(3) in order to study the asymptotic behavior of $u, v$ as $t \rightarrow \infty$, need to establish the uniform boundedness of global solutions; and 
(4) the global existence of solutions for the following full SKT system

$$
\begin{cases}u_{t}=d_{1} \Delta[(1+\alpha v+\gamma u) u]+a u(1-u-c v), & x \in \Omega, t>0 \\ v_{t}=d_{2} \Delta[(1+\beta u+\delta v) v]+b v(1-d u-v), & x \in \Omega, t>0 \\ \partial_{n} u=\partial_{n} v=0, & x \in \partial \Omega, t>0 \\ u(x, 0)=u_{0}(x), v(x, 0)=v_{0}(x), & x \in \Omega\end{cases}
$$

with $\alpha, \gamma, \beta, \delta>0$.

Very few global existence results for (1.7) are known. The main purpose of this article is to establish the uniform boundedness of global solutions for the system (1.7) in one space dimension. For convenience, we consider the following system

$$
\begin{cases}u_{t}=\left(d_{1} u+\alpha_{11} u^{2}+\alpha_{12} u v\right)_{x x}+u\left(a_{1}-b_{11} u-b_{12} v-e_{1} u v\right), & 0<x<1, t>0 \\ v_{t}=\left(d_{2} v+\alpha_{21} u v+\alpha_{22} v^{2}\right)_{x x}+v\left(a_{2}-b_{21} u-b_{22} v-e_{2} u v\right), & 0<x<1, t>0 \\ u_{x}(x, t)=v_{x}(x, t)=0, & x=0,1, t>0 \\ u(x, 0)=u_{0}(x), v(x, 0)=v_{0}(x), & 0<x<1\end{cases}
$$

We firstly investigate the global existence and the uniform boundedness of the solutions for (1.8), then prove the global asymptotic stability of the positive equilibrium $\left(u^{*}, v^{*}\right)$ of (1.8) by an important lemma from [21]. The proof is complete and complement to the uniform convergence theorems in [22-24].

It is obvious that $\left(u^{*}, v^{*}\right)$ is the unique positive equilibrium of the system (1.8) if (1.2) holds.

For simplicity, we denote $\|\cdot\|_{W_{p}^{k}(0,1)}$ by $|\cdot|_{k, p}$ and $\|\cdot\|_{L^{p}(0,1)}$ by $|\cdot|_{p}$. Our main results are as follows.

Theorem 1.1. Let $u_{0}, v_{0} \in W_{2}^{2}(0,1),(u, v)$ is the unique non-negative solution of system (1.2) in the maximal existence interval [0, $T$ ). Assume that

$$
8 \alpha_{11} \alpha_{21}>\alpha_{12}^{2}, \quad 8 \alpha_{22} \alpha_{12}>\alpha_{21}^{2} .
$$

Then there exist $t_{0}>0$ and positive constants $M, M^{\prime}$ which depend on $d_{i}, \alpha_{i j}, a_{i}, b_{i j}$, $e_{i}(i, j=1,2)$, such that

$$
\begin{aligned}
& \sup \left\{|u(\cdot, t)|_{1,2},|v(\cdot, t)|_{1,2}: t \in\left(t_{0}, T\right)\right\} \leq M^{\prime}, \\
& \max \left\{u(x, t), v(x, t):(x, t) \in[0,1] \times\left(t_{0}, T\right)\right\} \leq M,
\end{aligned}
$$

and $T=+\infty$. Moreover, in the case that $d_{1}, d_{2} \geq 1, d_{2} / d_{1} \in[\underline{d}, \bar{d}]$, where $\underline{d}$ and $\bar{d}$ are positive constants, $M^{\prime}, M$ depend on $\underline{d}, \bar{d}$, but do not depend on $d_{1}, d_{2}$.

Remark 1.1. Since the continuous embedding $H^{1}(\Omega) \otimes L^{\infty}(\Omega)$ holds only in one space dimension, we can only establish the uniform maximum-norm estimates about time for the solution in one space dimension.

Theorem 1.2 Assume that all conditions in Theorem 1.1 are satisfied. Assume further that,

$$
\begin{aligned}
& 4 d_{1} d_{2} u^{*} v^{*}>M^{2}\left(\alpha_{12} u^{*}+\alpha_{21} v^{*}\right)^{2} \\
& 4 b_{11} b_{22}>\left(b_{12}+b_{21}+e_{1} u^{*}+e_{2} v^{*}\right)^{2}
\end{aligned}
$$

and (1.2) hold, $M$ is given by (1.11). Then the unique positive equilibrium $\left(u^{*}, v^{*}\right)$ of (1.8) is globally asymptotically stable. 
Remark 1.2. The system (1.8) has no non-constant positive steady-state solution if all conditions of Theorem 1.2 hold.

\section{Global solutions}

In order to establish the uniform $W_{2}^{1}$-estimates of the solutions for system (1.2), the following Gagliardo-Nirenberg- type inequalities and the corresponding corollary play important roles (see [25]).

Theorem 2.1. Let $\Omega \subset \mathbb{R}^{n}$ be a bounded domain with $\partial \Omega\left\lfloor C^{m}\right.$. For every function $u \in W_{r}^{m}(\Omega), 1 \leq q, r \leq \infty$, the derivative $D^{j} u(0 \leq j<m)$ satisfies the inequality

$$
\left|D^{j} u\right|_{p} \leq C\left(\left|D^{m} u\right|_{r}^{a}|u|_{q}^{1-a}+|u|_{q}\right)
$$

provided one of the following three conditions is satisfied: (1) $r \leq q$, (2) $0<n(r-q) /$ $(m r q)<1$, or $(3) n(r-q) /(m r q)=1$ and $m-n / q$ is not a non-negative integer, where $1 / p$ $=j / n+a(1 / r-m / n)+(1-a) / q$ for all $a \in[j / m, 1)$, and the positive constant $C$ depends on $n, m, j, q, r, a$.

Corollary 1. There exists a positive constant $C$ such that

$$
\begin{aligned}
& |u|_{2} \leq C\left(\left|u_{x}\right|_{2}^{1 / 3}|u|_{1}^{2 / 3}+|u|_{1}\right), \quad \forall u \in W_{2}^{1}(0,1), \\
& |u|_{4} \leq C\left(\left|u_{x}\right|_{2}^{1 / 2}|u|_{1}^{1 / 2}+|u|_{1}\right), \quad \forall u \in W_{2}^{1}(0,1), \\
& \frac{|u|_{\frac{7}{2}}}{\frac{2}{2}} \leq C\left(\left|u_{x}\right|_{2}^{10 / 21}|u|_{1}^{11 / 21}+|u|_{1}\right), \quad \forall u \in W_{2}^{1}(0,1), \\
& \left|u_{x}\right|_{2} \leq C\left(\left|u_{x x}\right|_{2}^{3 / 5}|u|_{1}^{2 / 5}+|u|_{1}\right), \quad \forall u \in W_{2}^{2}(0,1) .
\end{aligned}
$$

Throughout this article, we always denote that $C$ is Sobolev embedding constant or other kind of universal constant, $A_{j}, B_{j}, C_{j}$ are some positive constants which depend only on $\alpha_{i j}, a_{i}, b_{i j}, e_{i}(i, j=1,2), K_{j}$ are positive constants depending on $d_{i}, \alpha_{i j}, a_{i}, b_{i j}, e_{i}$ $(i, j=1,2)$. When $d_{1}, d_{2} \geq 1, d_{2} / d_{1} \in[\underline{d}, \bar{d}], K_{j}$ depend on $\underline{d}, \bar{d}$, but do not depend on $d_{1}$ and $d_{2}$.

Proof of Theorem 1.1. Step 1, estimate $|u|_{1},|v|_{1}$.

Taking integration of the first, the first and the second equations in (1.8) over the domain $(0,1)$, respectively, we have

$$
\begin{aligned}
& \frac{d}{d t} \int_{0}^{1} u d x=\int_{0}^{1} u\left(a_{1}-b_{11} u-b_{12} v-e_{1} u v\right) d x \leq a_{1} \int_{0}^{1} u d x-b_{11}\left(\int_{0}^{1} u d x\right)^{2}, \\
& \frac{d}{d t} \int_{0}^{1} v d x=\int_{0}^{1} v\left(a_{2}-b_{21} u-b_{22} v-e_{2} u v\right) d x \leq a_{2} \int_{0}^{1} v d x-b_{22}\left(\int_{0}^{1} v d x\right)^{2} .
\end{aligned}
$$

So, there exists a positive constant $M_{0}$ which depends on $a_{i}, b_{i j}(i, j=1,2)$, such that

$$
\int_{0}^{1} u d x, \int_{0}^{1} v d x \leq M_{0}, \quad t \geq \tau_{0} .
$$


Moreover, there exists a positive constant $M_{0}^{\prime}$ which depends on $a_{i}, b_{i j}(i, j=1,2)$ and $L^{1}$-norm of $u_{0}, v_{0}$, such that

$$
\int_{0}^{1} u d x, \int_{0}^{1} v d x \leq M_{0}^{\prime}, \quad t \geq 0
$$

Step 2, estimate $|u|_{2},|v|_{2}$.

Multiplying the first two equations in system (1.2) by $u, v$, respectively, and integrating over $(0,1)$ we have

$$
\begin{aligned}
& \frac{1}{2} \frac{d}{d t} \int_{0}^{1} u^{2} d x \leq-d_{1} \int_{0}^{1} u_{x}^{2} d x-\int_{0}^{1}\left[\left(2 \alpha_{11} u+\alpha_{12} v\right) u_{x}^{2}+\alpha_{12} v_{x} u u_{x}\right] d x+a_{1} \int_{0}^{1} u^{2} d x, \\
& \frac{1}{2} \frac{d}{d t} \int_{0}^{1} v^{2} d x \leq-d_{2} \int_{0}^{1} v_{x}^{2} d x-\int_{0}^{1}\left[\left(\alpha_{21} u+2 \alpha_{22} v\right) v_{x}^{2}+\alpha_{21} u_{x} v v_{x}\right] d x+a_{2} \int_{0}^{1} v^{2} d x
\end{aligned}
$$

from which it follows that

$$
\begin{aligned}
\frac{1}{2} \frac{d}{d t} \int_{0}^{1}\left(u^{2}+v^{2}\right) d x & \leq-d \int_{0}^{1}\left(u_{x}^{2}+v_{x}^{2}\right) d x+a_{1} \int_{0}^{1} u^{2} d x+a_{2} \int_{0}^{1} v^{2} d x-\int_{0}^{1} q\left(u_{x}, v_{x}\right) d x \\
& \leq-d \int_{0}^{1}\left(u_{x}^{2}+v_{x}^{2}\right) d x+\left(a_{1}+a_{2}\right) \int_{0}^{1}\left(u^{2}+v^{2}\right) d x-\int_{0}^{1} q\left(u_{x}, v_{x}\right) d x,
\end{aligned}
$$

where $d=\min \left\{d_{1}, d_{2}\right\}$. Some tedious calculations yield that

$$
q\left(u_{x}, v_{x}\right)=\left(2 \alpha_{11} u+\alpha_{12} v\right) u_{x}^{2}+\left(\alpha_{12} u+\alpha_{21} v\right) u_{x} v_{x}+\left(\alpha_{21} u+2 \alpha_{22} v\right) v_{x}^{2}
$$

is positive definite quadratic form of $u_{x}, v_{x}$ if (1.9) holds. So (1.9) implies that

$$
\frac{1}{2} \frac{d}{d t} \int_{0}^{1}\left(u^{2}+v^{2}\right) d x \leq-d \int_{0}^{1}\left(u_{x}^{2}+v_{x}^{2}\right) d x+\left(a_{1}+a_{2}\right) \int_{0}^{1}\left(u^{2}+v^{2}\right) d x .
$$

Now, we proceed in the following two cases.

(i) $t \geq \tau_{0}$. The inequality (2.1) implies that $|u|_{2}^{6} \leq C\left(\left|u_{x}\right|_{2}^{2}|u|_{1}^{4}+|u|_{1}^{6}\right) \leq C M_{0}^{4}\left(\left|u_{x}\right|_{2}^{2}+M_{0}^{2}\right)$.

So we have $\int_{0}^{1} u_{x}^{2} d x \geq \frac{1}{C M_{0}^{4}}\left(\int_{0}^{1} u^{2} d x\right)^{3}-M_{0}^{2}$, and

$$
-\int_{0}^{1}\left(u_{x}^{2}+v_{x}^{2}\right) d x \leq-\frac{1}{9 C M_{0}^{4}}\left[\int_{0}^{1}\left(u^{2}+v^{2}\right) d x\right]^{3}+2 M_{0}^{2} .
$$

It follows from (2.5) and (2.6) that

$$
\frac{1}{2} \frac{d}{d t} \int_{0}^{1}\left(u^{2}+v^{2}\right) d x \leq d\left\{-C_{2}\left[\int_{0}^{1}\left(u^{2}+v^{2}\right) d x\right]^{3}+2 M_{0}^{2}+\frac{1}{d}\left(a_{1}+a_{2}\right) \int_{0}^{1}\left(u^{2}+v^{2}\right) d x\right\} .
$$

This means that there exist positive constants $\tau_{1}$ and $M_{1}$ depending on $d_{i}, a_{i}, b_{i j}(i, j=$ $1,2)$, such that 


$$
\int_{0}^{1} u^{2} d x, \int_{0}^{1} v^{2} d x \leq M_{1}, t \geq \tau_{1} .
$$

When $d \geq 1, M_{1}$ is independent of $d$ because the zero point of the right-hand side in (2.11) can be estimated by positive constants independent of $d$.

(ii) $t \geq 0$. Repeating estimates in (i) by (2.8)', we can obtain that there exists a positive constant $M_{1}^{\prime}$ depending on $d_{i}, a_{i}, b_{i j}(i, j=1,2)$ and the $L^{1}, L^{2}$-norm of $u_{0}, v_{0}$, such that

$$
\int_{0}^{1} u^{2} d x, \int_{0}^{1} v^{2} d x \leq M_{1}^{\prime}, \quad t \geq 0 .
$$

When $d \geq 1, M_{1}^{\prime}$ is independent of $d$.

Step 3, estimate $\left|u_{x}\right|_{2},\left|v_{x}\right|_{2}$.

Introducing the following scaling

$$
\tilde{u}=\frac{u}{d_{1}}, \tilde{v}=\frac{v}{d_{1}}, \tilde{t}=d_{1} t
$$

Denoting $\xi=d_{2} / d_{1}$, and using $u, v, t$ instead of $\tilde{u}, \tilde{v}, \tilde{t}$, respectively, then system (1.2) reduces to

$$
\begin{cases}u_{t}=P_{x x}+f(u, v), & 0<x<1, t>0, \\ v_{t}=Q_{x x}+g(u, v), & 0<x<1, t>0, \\ u_{x}(x, t)=v_{x}(x, t)=0, & x=0,1, t>0, \\ u(x, 0)=\tilde{u}_{0}(x), v(x, 0)=\tilde{v}_{0}(x), 0<x<1,\end{cases}
$$

where $P=u+\alpha_{11} u^{2}+\alpha_{12} u v, Q=\xi v+\alpha_{21} u v+\alpha_{22} v^{2}$, $g(u, v)=a_{2} d_{1}^{-1} v-b_{21} u v-b_{22} v^{2}-e_{2} d_{1} u v^{2}, g(u, v)=a_{2} d_{1}^{-1} v-b_{21} u v-b_{22} v^{2}-e_{2} d_{1} u v^{2}$.

We still proceed in the following two cases.

(i) $t \geq \tau_{1}^{*}=d_{1} \tau_{1}$. It is clear that

$$
\begin{gathered}
\int_{0}^{1} u d x, \int_{0}^{1} v d x \leq M_{0} d_{1}^{-1} \\
\int_{0}^{1} u^{2} d x, \int_{0}^{1} v^{2} d x \leq M_{1} d_{1}^{-2} \\
|P|_{1},|Q|_{1} \leq A_{1} K_{1} d_{1}^{-1}
\end{gathered}
$$

where $K_{1}=(1+\xi)+M_{1} d_{1}^{-1}$ and $A_{1}=\max \left\{M_{0}, \alpha_{11}+\alpha_{12}, \alpha_{21}+\alpha_{22}\right\}$.

Multiplying the first two equations in (2.11) by $P_{t}, Q_{t}$, integrating them over the domain $(0,1)$, respectively, and then adding up the two integration equalities, we have

$$
\begin{aligned}
\frac{1}{2} \bar{y}^{\prime}(t)= & -\int_{0}^{1} u_{t}^{2} d x-\xi \int_{0}^{1} v_{t}^{2} d x-\int_{0}^{1} q\left(u_{t}, v_{t}\right) d x \\
& +\int_{0}^{1}\left[\left(1+2 \alpha_{11} u+\alpha_{12} v\right) u_{t} f+\alpha_{12} u v_{t} f\right] d x+\int_{0}^{1}\left[\left(\xi+\alpha_{21} u+2 \alpha_{22} v\right) v_{t} g+\alpha_{21} v u_{t} g\right] d x
\end{aligned}
$$


where $\bar{\gamma}(t)=\int_{0}^{1}\left(P_{x}^{2}+Q_{x}^{2}\right) d x$. It is not hard verify by (1.9) that there exists a positive constant $C_{3}$ depending only on $\alpha_{i j}(i, j=1,2)$, such that

$$
q\left(u_{t}, v_{t}\right) \geq C_{3}(u+v)\left(u_{t}^{2}+v_{t}^{2}\right) .
$$

Thus,

$$
\begin{aligned}
\frac{1}{2} \bar{y}^{\prime}(t) \leq & -\int_{0}^{1} u_{t}^{2} d x-\xi \int_{0}^{1} v_{t}^{2} d x-C_{3} \int_{0}^{1}(u+v)\left(u_{t}^{2}+v_{t}^{2}\right) d x \\
& +\int_{0}^{1}\left[\left(1+2 \alpha_{11} u+\alpha_{12} v\right) u_{t} f+\alpha_{12} u v_{t} f\right] d x+\int_{0}^{1}\left[\left(\xi+\alpha_{21} u+2 \alpha_{22} v\right) v_{t} g+\alpha_{21} v u_{t} g\right] d x .
\end{aligned}
$$

Using Young inequality, Hölder inequality, and (2.12), we can obtain the following estimates

$$
\begin{aligned}
& \int_{0}^{1} u^{3} \mathrm{~d} x \leq\left(\int_{0}^{1} u^{7} \mathrm{~d} x\right)^{1 / 5}\left(\int_{0}^{1} u^{2} \mathrm{~d} x\right)^{4 / 5} \leq M_{1}^{4 / 5} d_{1}^{-8 / 5}\left(\int_{0}^{1} u^{7} \mathrm{~d} x\right)^{1 / 5}, \\
& \left.\int_{0}^{1} u^{4} \mathrm{~d} x \leq\left(\int_{0}^{1} u^{7} \mathrm{~d} x\right)^{2 / 5}\left(\int_{0}^{1} u^{2} \mathrm{~d} x\right)^{3 / 5} \leq M_{1}^{3 / 5} d_{1}^{-6 / 5}\left(\int_{0}^{1} u^{7} \mathrm{~d} x\right)\right)^{2 / 5}, \\
& \int_{0}^{1} u^{5} \mathrm{~d} x \leq\left(\int_{0}^{1} u^{7} \mathrm{~d} x\right)^{3 / 5}\left(\int_{0}^{1} u^{2} \mathrm{~d} x\right)^{2 / 5} \leq M_{1}^{2 / 5} d_{1}^{-4 / 5}\left(\int_{0}^{1} u^{7} \mathrm{~d} x\right)^{3 / 5}, \\
& \int_{0}^{1} u^{6} \mathrm{~d} x \leq\left(\int_{0}^{1} u^{7} \mathrm{~d} x\right)^{4 / 5}\left(\int_{0}^{1} u^{2} \mathrm{~d} x\right)^{1 / 5} \leq M_{1}^{1 / 5} d_{1}^{-2 / 5}\left(\int_{0}^{1} u^{7} \mathrm{~d} x\right)^{4 / 5}, \\
& \int_{0}^{1} u^{2} v \mathrm{~d} x \leq\left(\int_{0}^{1} u^{7} \mathrm{~d} x\right)^{1 / 5}\left(\int_{0}^{1} u^{2} \mathrm{~d} x\right)^{3 / 10}\left(\int_{0}^{1} v^{2} \mathrm{~d} x\right)^{1 / 2} \leq M_{1}^{4 / 5} d_{1}^{-8 / 5}\left(\int_{0}^{1} u^{7} \mathrm{~d} x\right)^{1 / 5}, \\
& \int_{0}^{1} u^{3} v \mathrm{~d} x \leq\left(\int_{0}^{1} u^{7} \mathrm{~d} x\right)^{2 / 5}\left(\int_{0}^{1} u^{2} \mathrm{~d} x\right)^{1 / 10}\left(\int_{0}^{1} v^{2} \mathrm{~d} x\right)^{1 / 2} \leq M_{1}^{3 / 5} d_{1}^{-6 / 5}\left(\int_{0}^{1} u^{7} \mathrm{~d} x\right)^{2 / 5}, \\
& \int_{0}^{1} u^{4} v \mathrm{~d} x \leq \frac{4}{5} \int_{0}^{1} u^{5} \mathrm{~d} x+\frac{1}{5} \int_{0}^{1} v^{5} \mathrm{~d} x \leq \frac{4}{5} M_{1}^{2 / 5} d_{1}^{-4 / 5}\left[\left(\int_{0}^{1} u^{7} \mathrm{~d} x\right)^{3 / 5}+\left(\int_{0}^{1} v^{7} \mathrm{~d} x\right)^{3 / 5}\right], \\
& \int_{0}^{1} u^{2} v^{2} \mathrm{~d} x \leq \frac{1}{2}\left(\int_{0}^{1} u^{4} \mathrm{~d} x+\int_{0}^{1} v^{4} \mathrm{~d} x\right) \leq \frac{1}{2} M_{1}^{3 / 5} d_{1}^{-4 / 5}\left[\left(\int_{0}^{1} u^{7} \mathrm{~d} x\right)^{2 / 5}+\left(\int_{0}^{1} v^{7} \mathrm{~d} x\right)^{2 / 5}\right], \\
& \int_{0}^{1} u^{3} v^{2} \mathrm{~d} x \leq \frac{3}{5} \int_{0}^{1} u^{5} \mathrm{~d} x+\frac{2}{5} \int_{0}^{1} v^{5} \mathrm{~d} x \leq \frac{3}{5} M_{1}^{2 / 5} d_{1}^{-4 / 5}\left[\left(\int_{0}^{1} u^{7} \mathrm{~d} x\right)^{3 / 5}+\left(\int_{0}^{1} v^{7} \mathrm{~d} x\right)^{3 / 5}\right], \\
& \int_{0}^{1} u^{4} v^{2} \mathrm{~d} x \leq \frac{2}{3} \int_{0}^{1} u^{6} \mathrm{~d} x+\frac{1}{3} \int_{0}^{1} v^{6} \mathrm{~d} x \leq \frac{2}{3} M_{1}^{1 / 5} d_{1}^{-4 / 5}\left[\left(\int_{0}^{1} u^{7} \mathrm{~d} x\right)^{4 / 5}+\left(\int_{0}^{1} v^{7} \mathrm{~d} x\right)^{4 / 5}\right], \\
& \int_{0}^{1} u^{4} v^{3} \mathrm{~d} x \leq \frac{4}{7} \int_{0}^{1} u^{7} \mathrm{~d} x+\frac{3}{7} \int_{0}^{1} v^{7} \mathrm{~d} x \leq \frac{4}{7}\left(\int_{0}^{1} u^{7} \mathrm{~d} x+\int_{0}^{1} v^{7} \mathrm{~d} x\right), \\
& \int_{0}^{1} u^{5} v^{2} \mathrm{~d} x \leq \frac{5}{7} \int_{0}^{1} u^{7} \mathrm{~d} x+\frac{2}{7} \int_{0}^{1} v^{7} \mathrm{~d} x \leq \frac{5}{7}\left(\int_{0}^{1} u^{7} \mathrm{~d} x+\int_{0}^{1} v^{7} \mathrm{~d} x\right) .
\end{aligned}
$$


Liao Advances in Difference Equations 2012, 2012:89

Page 9 of 16

Applying the above estimates and Gagliardo-Nirenberg-type inequalities to the terms on the right-hand side of (2.13), we have

$$
\begin{aligned}
& -\int_{0}^{1} u_{t}^{2} d x \leq-\frac{1}{2} \int_{0}^{1} P_{x x}^{2} d x+\int_{0}^{1} f^{2} d x \\
& -\xi \int_{0}^{1} v_{t}^{2} d x \leq-\frac{\xi}{2} \int_{0}^{1} Q_{x x}^{2} d x+\xi \int_{0}^{1} g^{2} d x \\
& \int_{0}^{1} f^{2} d x \leq \int_{0}^{1}\left(a_{1}^{2} d_{1}^{-2} u^{2}+b_{11}^{2} u^{4}+b_{12}^{2} u^{2} v^{2}+e_{1}^{2} d_{1}^{2} u^{4} v^{2}+2 b_{11} b_{12} u^{3} v+2 b_{11} e_{1} d_{1} u^{4} v+2 b_{12} e_{1} d_{1} u^{3} v^{2}\right) d x \\
& \leq a_{1}^{2} M_{1} d_{1}^{-4}+\left(b_{11}^{2}+\frac{1}{2} b_{12}^{2}+2 b_{11} b_{12}\right) M_{1}^{3 / 5} d_{1}^{-6 / 5}\left[\left(\int_{0}^{1} u^{7} \mathrm{~d} x\right)^{2 / 5}+\left(\int_{0}^{1} v^{7} \mathrm{~d} x\right)^{2 / 5}\right] \\
& +\left(\frac{8}{5} b_{11}+\frac{6}{5} b_{12}\right) e_{1} M_{1}^{2 / 5} d_{1}^{1 / 5}\left[\left(\int_{0}^{1} u^{7} \mathrm{~d} x\right)^{3 / 5}+\left(\int_{0}^{1} v^{7} \mathrm{~d} x\right)^{3 / 5}\right] \\
& +\frac{2}{3} e_{1}^{2} M_{1}^{1 / 5} d_{1}^{8 / 5}\left[\left(\int_{0}^{1} u^{7} \mathrm{~d} x\right)^{4 / 5}+\left(\int_{0}^{1} v^{7} \mathrm{~d} x\right)^{4 / 5}\right]
\end{aligned}
$$

$$
\begin{aligned}
\xi \int_{0}^{1} g^{2} d x & \leq \xi \int_{0}^{1}\left(a_{2}^{2} d_{1}^{-2} v^{2}+b_{21}^{2} u^{2} v^{2}+b_{22}^{2} v^{4}+e_{2}^{2} d_{1}^{2} u^{2} v^{4}+2 b_{21} b_{22} u v^{3}+2 b_{21} e_{2} d_{1} u^{2} v^{3}+2 b_{22} e_{2} d_{1} u v^{4}\right) d x \\
& \leq \xi a_{2}^{2} M_{1} d_{1}^{-4}+\xi\left(b_{22}^{2}+\frac{1}{2} b_{21}^{2}+2 b_{21} b_{22}\right) M_{1}^{3 / 5} d_{1}^{-6 / 5}\left[\left(\int_{0}^{1} u^{7} \mathrm{~d} x\right)^{2 / 5}+\left(\int_{0}^{1} v^{7} \mathrm{~d} x\right)^{2 / 5}\right] \\
& +2 \xi\left(\frac{3}{5} b_{21}+\frac{4}{5} b_{22}\right) e_{2} M_{1}^{2 / 5} d_{1}^{1 / 5}\left[\left(\int_{0}^{1} u^{7} \mathrm{~d} x\right)^{3 / 5}+\left(\int_{0}^{1} v^{7} \mathrm{~d} x\right)^{3 / 5}\right] \\
& +\frac{2}{3} \xi e_{2}^{2} M_{1}^{1 / 5} d_{1}^{8 / 5}\left[\left(\int_{0}^{1} u^{7} \mathrm{~d} x\right)^{4 / 5}+\left(\int_{0}^{1} v^{7} \mathrm{~d} x\right)^{4 / 5}\right]
\end{aligned}
$$

and

$$
\begin{aligned}
-\int_{0}^{1} u_{t}^{2} d x-\xi \int_{0}^{1} v_{1}^{2} d x \leq & -\frac{1}{2} \int_{0}^{1} P_{x x}^{2} d x-\frac{\xi}{2} \int_{0}^{1} Q_{x x}^{2} d x+\left(a_{1}^{2}+\xi a_{2}^{2}\right) M_{1} d_{1}^{-4} \\
& +\left[\left(b_{11}^{2}+\frac{1}{2} b_{12}^{2}+2 b_{11} b_{12}\right)+\xi\left(b_{22}^{2}+\frac{1}{2} b_{21}^{2}+2 b_{21} b_{22}\right)\right] M_{1}^{3 / 5} d_{1}^{-6 / 5}\left[\left(\int_{0}^{1} u^{7} \mathrm{~d} x\right)^{2 / 5}+\left(\int_{0}^{1} v^{7} \mathrm{dx}\right)^{2 / 5}\right] \\
& +\left[\left(\frac{8}{5} b_{11}+\frac{6}{5} b_{12}\right) e_{1}+2 \xi\left(\frac{3}{5} b_{21}+\frac{4}{5} b_{22}\right) e_{2}\right] M_{1}^{2 / 5} d_{1}^{1 / 5}\left[\left(\int_{0}^{1} u^{7} \mathrm{~d} x\right)^{3 / 5}+\left(\int_{0}^{1} v^{7} \mathrm{~d} x\right)^{3 / 5}\right] \\
& +\frac{2}{3}\left(e_{1}^{2}+\xi e_{2}^{2}\right) M_{1}^{1 / 5} d_{1}^{8 / 5}\left[\left(\int_{0}^{1} u^{7} \mathrm{~d} x\right)^{4 / 5}+\left(\int_{0}^{1} v^{7} \mathrm{~d} x\right)^{4 / 5}\right] .
\end{aligned}
$$


Similarly, we can obtain

$$
\begin{aligned}
& \int_{0}^{1} u_{t} f d x \leq \int_{0}^{1} u_{t}\left(a_{1} d_{1}^{-1} u+b_{11} u^{2}+b_{12} u v+e_{1} d_{1} u^{2} v\right) d x \\
& \leq \frac{a_{1}^{2} d_{1}^{-2}}{2 \varepsilon} \int_{0}^{1} u d x+\frac{\varepsilon}{2} \int_{0}^{1} u u_{t}^{2} d x+\frac{b_{11}^{2}}{2 \varepsilon} \int_{0}^{1} u^{3} d x+\frac{\varepsilon}{2} \int_{0}^{1} u u_{t}^{2} d x \\
& +\frac{b_{12}^{2}}{2 \varepsilon} \int_{0}^{1} u v^{2} d x+\frac{\varepsilon}{2} \int_{0}^{1} u u_{t}^{2} d x+\frac{\left(e_{1} d_{1}\right)^{2}}{2 \varepsilon} \int_{0}^{1} u^{3} v^{2} d x+\frac{\varepsilon}{2} \int_{0}^{1} u u_{t}^{2} d x \\
& \leq \frac{a_{1}^{2}}{2 \varepsilon} M_{0} d_{1}^{-3}+\frac{b_{11}^{2}+b_{12}^{2}}{2 \varepsilon} M_{1}^{4 / 5} d_{1}^{-8 / 5}\left[\left(\int_{0}^{1} u^{7} \mathrm{~d} x\right)^{1 / 5}+\left(\int_{0}^{1} v^{7} \mathrm{~d} x\right)^{1 / 5}\right] \\
& +\frac{3 e_{1}^{2}}{10 \varepsilon} M_{1}^{2 / 5} d_{1}^{6 / 5}\left[\left(\int_{0}^{1} u^{7} \mathrm{~d} x\right)^{3 / 5}+\left(\int_{0}^{1} v^{7} \mathrm{~d} x\right)^{3 / 5}\right]+2 \varepsilon \int_{0}^{1} u u_{t}^{2} d x \\
& 2 \alpha_{11} \int_{0}^{1} u u_{t} f d x \leq 2 \alpha_{11} \int_{0}^{1} u u_{t}\left(a_{1} d_{1}^{-1} u+b_{11} u^{2}+b_{12} u v+e_{1} d_{1} u^{2} v\right) d x \\
& \leq \frac{\alpha_{11}^{2} a_{1}^{2} d_{1}^{-2}}{\varepsilon} \int_{0}^{1} u^{3} d x+\varepsilon \int_{0}^{1} u u_{t}^{2} d x+\frac{\alpha_{11}^{2} b_{11}^{2}}{\varepsilon} \int_{0}^{1} u^{5} d x+\varepsilon \int_{0}^{1} u u_{t}^{2} d x \\
& +\frac{\alpha_{11}^{2} b_{12}^{2}}{\varepsilon} \int_{0}^{1} u^{3} v^{2} d x+\varepsilon \int_{0}^{1} u u_{t}^{2} d x+\frac{\alpha_{11}^{2} e_{1}^{2} d_{1}^{2}}{\varepsilon} \int_{0}^{1} u^{5} v^{2} d x+\varepsilon \int_{0}^{1} u u_{t}^{2} d x \\
& \leq \frac{\alpha_{11}^{2} a_{1}^{2}}{\varepsilon} M_{1}^{4 / 5} d_{1}^{-18 / 5}\left(\int_{0}^{1} u^{7} d x\right)^{1 / 5}+\frac{\alpha_{11}^{2}\left(b_{11}^{2}+b_{12}^{2}\right)}{\varepsilon} M_{1}^{2 / 5} d_{1}^{-4 / 5}\left[\left(\int_{0}^{1} u^{7} \mathrm{~d} x\right)^{3 / 5}+\left(\int_{0}^{1} v^{7} \mathrm{~d} x\right)^{3 / 5}\right] \\
& +\frac{5 \alpha_{11}^{2} e_{1}^{2}}{7 \varepsilon} d_{1}^{2}\left(\int_{0}^{1} u^{7} \mathrm{~d} x+\int_{0}^{1} v^{7} \mathrm{~d} x\right)+2 \varepsilon \int_{0}^{1} u u_{t}^{2} d x \\
& \alpha_{12} \int_{0}^{1} v u_{t} f d x \leq \alpha_{12} \int_{0}^{1} v u_{t}\left(a_{1} d_{1}^{-1} u+b_{11} u^{2}+b_{12} u v+e_{1} d_{1} u^{2} v\right) d x \\
& \leq \frac{\alpha_{12}^{2} a_{1}^{2} d_{1}^{-2}}{2 \varepsilon} \int_{0}^{1} u v^{2} d x+\frac{\varepsilon}{2} \int_{0}^{1} u u_{t}^{2} d x+\frac{\alpha_{12}^{2} b_{11}^{2}}{2 \varepsilon} \int_{0}^{1} u^{3} v^{2} d x+\frac{\varepsilon}{2} \int_{0}^{1} u u_{t}^{2} d x \\
& +\frac{\alpha_{12}^{2} b_{12}^{2}}{2 \varepsilon} \int_{0}^{1} u v^{4} d x+\frac{\varepsilon}{2} \int_{0}^{1} u u_{t}^{2} d x+\frac{\alpha_{12}^{2} e_{1}^{2} d_{1}^{2}}{2 \varepsilon} \int_{0}^{1} u^{3} v^{4} d x+\frac{\varepsilon}{2} \int_{0}^{1} u u_{t}^{2} d x \\
& \leq \frac{\alpha_{12}^{2} a_{1}^{2}}{2 \varepsilon} M_{1}^{4 / 5} d_{1}^{-18 / 5}\left(\int_{0}^{1} v^{7} d x\right)^{1 / 5}+\frac{\alpha_{12}^{2}\left(b_{11}^{2}+b_{12}^{2}\right)}{2 \varepsilon} M_{1}^{2 / 5} d_{1}^{-4 / 5}\left[\left(\int_{0}^{1} u^{7} \mathrm{~d} x\right)^{3 / 5}+\left(\int_{0}^{1} v^{7} \mathrm{~d} x\right)^{3 / 5}\right] \\
& +\frac{2 \alpha_{12}^{2} e_{1}^{2}}{7 \varepsilon} d_{1}^{2}\left(\int_{0}^{1} u^{7} \mathrm{~d} x+\int_{0}^{1} v^{7} \mathrm{~d} x\right)+2 \varepsilon \int_{0}^{1} u u_{t}^{2} d x
\end{aligned}
$$


Jiao Advances in Difference Equations 2012, 2012:89

Page 11 of 16

http://www.advancesindifferenceequations.com/content/2012/1/89

$$
\begin{aligned}
\alpha_{12} \int_{0}^{1} u v_{t} f d x \leq & \alpha_{12} \int_{0}^{1} u v_{t}\left(a_{1} d_{1}^{-1} u+b_{11} u^{2}+b_{12} u v+e_{1} d_{1} u^{2} v\right) d x \\
\leq & \frac{\alpha_{12}^{2} a_{1}^{2} d_{1}^{-2}}{2 \varepsilon} \int_{0}^{1} u^{3} d x+\frac{\varepsilon}{2} \int_{0}^{1} u v_{t}^{2} d x+\frac{\alpha_{12}^{2} b_{11}^{2}}{2 \varepsilon} \int_{0}^{1} u^{5} d x+\frac{\varepsilon}{2} \int_{0}^{1} u v_{t}^{2} d x \\
& +\frac{\alpha_{12}^{2} b_{12}^{2}}{2 \varepsilon} \int_{0}^{1} u^{3} v^{2} d x+\frac{\varepsilon}{2} \int_{0}^{1} u v_{t}^{2} d x+\frac{\alpha_{12}^{2} e_{1}^{2} d_{1}^{2}}{2 \varepsilon} \int_{0}^{1} u^{5} v^{2} d x+\frac{\varepsilon}{2} \int_{0}^{1} u v_{t}^{2} d x \\
\leq & \frac{\alpha_{12}^{2} a_{1}^{2}}{2 \varepsilon} M_{1}^{4 / 5} d_{1}^{-18 / 5}\left(\int_{0}^{1} u^{7} d x\right)^{1 / 5}+\frac{\alpha_{12}^{2}\left(b_{11}^{2}+b_{12}^{2}\right)}{2 \varepsilon} M_{1}^{2 / 5} d_{1}^{-4 / 5}\left[\left(\int_{0}^{1} u^{7} \mathrm{~d} x\right)^{3 / 5}+\left(\int_{0}^{1} v^{7} \mathrm{~d} x\right)^{3 / 5}\right] \\
& +\frac{5 \alpha_{12}^{2} e_{1}^{2}}{14 \varepsilon} d_{1}^{2}\left(\int_{0}^{1} u^{7} \mathrm{~d} x+\int_{0}^{1} v^{7} \mathrm{~d} x\right)+2 \varepsilon \int_{0}^{1} u v_{t}^{2} d x
\end{aligned}
$$

$$
\begin{aligned}
\xi \int_{0}^{1} v_{t} g d x \leq & \xi \int_{0}^{1} v_{t}\left(a_{2} d_{1}^{-1} v+b_{21} u v+b_{22} v^{2}+e_{2} d_{1} u v^{2}\right) d x \\
\leq & \frac{\xi^{2} a_{2}^{2} d_{1}^{-2}}{2 \varepsilon} \int_{0}^{1} v d x+\frac{\varepsilon}{2} \int_{0}^{1} v v_{t}^{2} d x+\frac{\xi^{2} b_{21}^{2}}{2 \varepsilon} \int_{0}^{1} u^{2} v d x+\frac{\varepsilon}{2} \int_{0}^{1} v v_{t}^{2} d x \\
& +\frac{\xi^{2} b_{22}^{2}}{2 \varepsilon} \int_{0}^{1} v^{3} d x+\frac{\varepsilon}{2} \int_{0}^{1} v v_{t}^{2} d x+\frac{\xi^{2} e_{2}^{2} d_{1}^{2}}{2 \varepsilon} \int_{0}^{1} u^{2} v^{3} d x+\frac{\varepsilon}{2} \int_{0}^{1} v v_{t}^{2} d x \\
\leq & \frac{\xi^{2} a_{2}^{2}}{2 \varepsilon} M_{0} d_{1}^{-3}+\frac{\xi^{2}\left(b_{21}^{2}+b_{22}^{2}\right)}{2 \varepsilon} M_{1}^{4 / 5} d_{1}^{-8 / 5}\left[\left(\int_{0}^{1} u^{7} \mathrm{~d} x\right)^{1 / 5}+\left(\int_{0}^{1} v^{7} \mathrm{~d} x\right)^{1 / 5}\right] \\
& +\frac{3 \xi^{2} e_{2}^{2}}{10 \varepsilon} M_{1}^{2 / 5} d_{1}^{6 / 5}\left[\left(\int_{0}^{1} u^{7} \mathrm{~d} x\right)^{3 / 5}+\left(\int_{0}^{1} v^{7} \mathrm{~d} x\right)^{3 / 5}\right]+2 \varepsilon \int_{0}^{1} v v_{t}^{2} d x,
\end{aligned}
$$

$$
\begin{aligned}
\alpha_{21} \int_{0}^{1} u v_{t} g d x \leq & \alpha_{21} \int_{0}^{1} u v_{t}\left(a_{2} d_{1}^{-1} v+b_{21} u v+b_{22} v^{2}+e_{2} d_{1} u v^{2}\right) d x \\
\leq & \frac{\alpha_{21}^{2} a_{2}^{2} d_{1}^{-2}}{2 \varepsilon} \int_{0}^{1} u^{2} v d x+\frac{\varepsilon}{2} \int_{0}^{1} v v_{t}^{2} d x+\frac{\alpha_{21}^{2} b_{21}^{2}}{2 \varepsilon} \int_{0}^{1} u^{4} v d x+\frac{\varepsilon}{2} \int_{0}^{1} v v_{t}^{2} d x \\
& +\frac{\alpha_{21}^{2} b_{22}^{2}}{2 \varepsilon} \int_{0}^{1} u^{2} v^{3} d x+\frac{\varepsilon}{2} \int_{0}^{1} v v_{t}^{2} d x+\frac{\alpha_{21}^{2} e_{2}^{2} d_{1}^{2}}{2 \varepsilon} \int_{0}^{1} u^{4} v^{3} d x+\frac{\varepsilon}{2} \int_{0}^{1} v v_{t}^{2} d x \\
\leq & \frac{\alpha_{21}^{2} a_{2}^{2}}{2 \varepsilon} M_{1}^{4 / 5} d_{1}^{-18 / 5}\left(\int_{0}^{1} u^{7} d x\right)^{1 / 5}+\frac{\alpha_{21}^{2}\left(b_{21}^{2}+b_{22}^{2}\right)}{2 \varepsilon} M_{1}^{2 / 5} d_{1}^{-4 / 5}\left[\left(\int_{0}^{1} u^{7} \mathrm{~d} x\right)^{3 / 5}+\left(\int_{0}^{1} v^{7} \mathrm{~d} x\right)^{3 / 5}\right] \\
& +\frac{5 \alpha_{21}^{2} e_{2}^{2}}{14 \varepsilon} d_{1}^{2}\left(\int_{0}^{1} u^{7} \mathrm{~d} x+\int_{0}^{1} v^{7} \mathrm{~d} x\right)+2 \varepsilon \int_{0}^{1} v v_{t}^{2} d x
\end{aligned}
$$




$$
\begin{aligned}
& 2 \alpha_{22} \int_{0}^{1} v v_{t} g d x \leq 2 \alpha_{22} \int_{0}^{1} v v_{t}\left(a_{2} d_{1}^{-1} v+b_{21} u v+b_{22} v^{2}+e_{2} d_{1} u v^{2}\right) d x \\
& \leq \frac{\alpha_{22}^{2} a_{2}^{2} d_{1}^{-2}}{\varepsilon} \int_{0}^{1} v^{3} d x+\varepsilon \int_{0}^{1} v v_{t}^{2} d x+\frac{\alpha_{22}^{2} b_{21}^{2}}{\varepsilon} \int_{0}^{1} u^{2} v^{3} d x+\varepsilon \int_{0}^{1} v v_{t}^{2} d x \\
&+\frac{\alpha_{22}^{2} b_{22}^{2}}{\varepsilon} \int_{0}^{1} v^{5} d x+\varepsilon \int_{0}^{1} v v_{t}^{2} d x+\frac{\alpha_{22}^{2} e_{2}^{2} d_{1}^{2}}{\varepsilon} \int_{0}^{1} u^{2} v^{5} d x+\varepsilon \int_{0}^{1} v v_{t}^{2} d x \\
& \leq \frac{\alpha_{22}^{2} a_{2}^{2}}{\varepsilon} M_{1}^{4 / 5} d_{1}^{-18 / 5}\left(\int_{0}^{1} v^{7} d x\right)^{1 / 5}+\frac{\alpha_{22}^{2}\left(b_{21}^{2}+b_{22}^{2}\right)}{\varepsilon} M_{1}^{2 / 5} d_{1}^{-4 / 5}\left[\left(\int_{0}^{1} u^{7} \mathrm{~d} x\right)^{3 / 5}+\left(\int_{0}^{1} v^{7} \mathrm{~d} x\right)^{3 / 5}\right] \\
&+\frac{5 \alpha_{22}^{2} e_{2}^{2}}{7 \varepsilon} d_{1}^{2}\left(\int_{0}^{1} u^{7} \mathrm{~d} x+\int_{0}^{1} v^{7} \mathrm{~d} x\right)+2 \varepsilon \int_{0}^{1} v v_{t}^{2} d x \\
& \alpha_{21} \int_{0}^{1} v u_{t} g d x \leq \alpha_{21} \int_{0}^{1} v u_{t}\left(a_{2} d_{1}^{-1} v+b_{21} u v+b_{22} v^{2}+e_{2} d_{1} u v^{2}\right) d x \\
& \leq \frac{\alpha_{21}^{2} a_{2}^{2} d_{1}^{-2}}{2 \varepsilon} \int_{0}^{1} v^{3} d x+\frac{\varepsilon}{2} \int_{0}^{1} v u_{t}^{2} d x+\frac{\alpha_{21}^{2} b_{21}^{2}}{2 \varepsilon} \int_{0}^{1} u^{2} v^{3} d x+\frac{\varepsilon}{2} \int_{0}^{1} v u_{t}^{2} d x \\
&+\frac{\alpha_{21}^{2} b_{22}^{2}}{2 \varepsilon} \int_{0}^{1} v^{5} d x+\frac{5}{2} \int_{0}^{1} v u_{t}^{2} d x+\frac{\alpha_{21}^{2} e_{2}^{2} d_{1}^{2}}{2 \varepsilon} \int_{0}^{1} u^{2} v^{5} d x+\frac{\varepsilon}{2} \int_{0}^{1} v u_{t}^{2} d x \\
& \leq \frac{\alpha_{21}^{2} a_{2}^{2}}{2 \varepsilon} M_{1}^{4 / 5} d_{1}^{-18 / 5}\left(\int_{0}^{1} v^{7} u^{7} \mathrm{~d} x+\int_{0}^{1} v^{1 / 5} \mathrm{~d} x\right)+2 \varepsilon \int_{0}^{1} v v_{t}^{2} d x . \\
& 2 \varepsilon \alpha_{21}^{2}\left(b_{21}^{2}+b_{22}^{2}\right) \\
& M_{1}^{2 / 5} d_{1}^{-4 / 5}\left[\left(\int_{0}^{1} u^{7} \mathrm{~d} x\right)^{3 / 5}+\left(\int_{0}^{1} v^{7} \mathrm{~d} x\right)^{3 / 5}\right]
\end{aligned}
$$

By the above inequalities and the condition (1.9), we have

$$
\begin{aligned}
& \int_{0}^{1}\left[\left(1+2 \alpha_{11} u+\alpha_{12} v\right) u_{t} f+\alpha_{12} u v_{t} f\right] d x+\int_{0}^{1}\left[\left(\xi+\alpha_{21} u+2 \alpha_{22} v\right) v_{t} g+\alpha_{21} v u_{t} g\right] d x \\
& \leq \bar{\lambda} \varepsilon \int_{0}^{1}(u+v)\left(u_{t}^{2}+v_{t}^{2}\right) d x+\frac{C_{4}}{\varepsilon}\left(1+\xi^{2}\right) M_{0} d_{1}^{-3}+\frac{C_{5}}{\varepsilon}\left(1+\xi^{2}+d_{1}^{-2}\right) M_{1}^{4 / 5} d_{1}^{-8 / 5}\left[\int_{0}^{1}\left(u^{7}+v^{7}\right) d x\right]^{1 / 5} \\
& \quad+\frac{C_{5}}{\varepsilon}\left(1+\xi^{2}+d_{1}^{-2}\right) M_{1}^{2 / 5} d_{1}^{6 / 5}\left[\int_{0}^{1}\left(u^{7}+v^{7}\right) d x\right]^{1 / 5}+\frac{C_{6}}{\varepsilon} d_{1}^{2} \int_{0}^{1}\left(u^{7}+v^{7}\right) d x
\end{aligned}
$$

where $\bar{\lambda}$ is a constant. Choose a small enough positive number $\varepsilon\left(\alpha_{i j}, a_{i}, b_{i j}, e_{i}\right)(i, j=$ 1, 2), such that $\bar{\lambda} \varepsilon<C_{3}$.

Substituting inequalities (2.15) and (2.16) into (2.13), one can obtain

$$
\frac{1}{2} \bar{y}^{\prime}(t) \leq-\frac{1}{2} \int_{0}^{1} P_{x x}^{2} d x-\frac{\xi}{2} \int_{0}^{1} Q_{x x}^{2} d x+B_{1} K_{2} d_{1}^{-3}+B_{2} d_{1}^{2} Y+B_{3} K_{3} d_{1}^{6 / 5} Y^{3 / 5}+B_{4} K_{4} d_{1}^{-8 / 5} Y^{1 / 5}
$$

where $\quad Y=\int_{0}^{1}\left(u^{7}+v^{7}\right) d x$,

$$
K_{2}=\left(1+\xi^{2}\right) M_{0}
$$$$
K_{3}=\left(1+\xi^{2}+d_{1}^{-2}\right) M_{1}^{2 / 5},
$$

$K_{4}=\left(1+\xi^{2}+d_{1}^{-2}\right) M_{1}^{4 / 5}$. 
Clearly,

$$
P \geq \alpha_{11} u^{2}, \quad Q \geq \alpha_{22} v^{2} .
$$

It follows from (2.12) and (2.3) to functions $P, Q$ that

$$
\begin{aligned}
& Y \leq B_{5} \int_{0}^{1}\left(P^{7 / 2}+Q^{7 / 2}\right) d x \leq B_{6} K_{1}^{11 / 6} d_{1}^{-11 / 6} \bar{y}^{5 / 6}+B_{6} K_{1}^{7 / 2} d_{1}^{-7 / 2} \\
& Y^{1 / 5} \leq B_{7} K_{1}^{11 / 30} d_{1}^{-11 / 30} \bar{y}^{1 / 6}+B_{7} K_{1}^{7 / 10} d_{1}^{-7 / 10} \\
& Y^{3 / 5} \leq B_{8} K_{1}^{11 / 10} d_{1}^{-11 / 10} \bar{y}^{1 / 2}+B_{8} K_{1}^{21 / 10} d_{1}^{-21 / 10}
\end{aligned}
$$

Moreover, one can obtain by (2.4) and (2.12)

$$
-\frac{1}{2} \int_{0}^{1} P_{x x}^{2} d x-\frac{\xi}{2} \int_{0}^{1} Q_{x x}^{2} d x \leq-B_{9} \min \{1, \xi\} K_{1}^{-4 / 3} d_{1}^{4 / 3} \bar{y}^{5 / 3}+(1+\xi) K_{1}^{2} d_{1}^{-2} .
$$

Combining (2.16), (2.18), and (2.19), we have

$$
\begin{aligned}
\frac{1}{2} \bar{y}^{\prime}(t) \leq & -A_{1} \min \{1, \xi\} K_{1}^{-4 / 3} d_{1}^{4 / 3} \bar{y}^{5 / 3} \\
& +A_{2}\left[(1+\xi) K_{1}^{2} d_{1}^{-2}+K_{2} d_{1}^{-3}+K_{1}^{7 / 2} d_{1}^{-3 / 2}+K_{1}^{21 / 10} K_{3} d_{1}^{-9 / 10}+K_{1}^{7 / 10} K_{4} d_{1}^{-23 / 10}\right] \\
& +A_{3} K_{1}^{11 / 6} d_{1}^{1 / 6} \bar{y}^{5 / 6}+A_{4} K_{1}^{11 / 10} K_{3} d_{1}^{1 / 10} \bar{y}^{1 / 2}+A_{5} K_{1}^{11 / 30} K_{4} d_{1}^{-59 / 30} \bar{y}^{1 / 6} .
\end{aligned}
$$

Multiplying inequality $(2.20)$ by $d_{1}^{2}$, we have

$$
\begin{aligned}
\frac{1}{2} \gamma^{\prime}(t) \leq & -A_{1} \min \{1, \xi\} K_{1}^{-4 / 3} \gamma^{5 / 3} \\
& +A_{2}\left[(1+\xi) K_{1}^{2}+K_{2} d_{1}^{-1}+K_{1}^{7 / 2} d_{1}^{1 / 2}+K_{1}^{21 / 10} K_{3} d_{1}^{11 / 10}+K_{1}^{7 / 10} K_{4} d_{1}^{-3 / 10}(2.21)\right. \\
& +A_{3} K_{1}^{11 / 6} d_{1}^{1 / 2} \gamma^{5 / 6}+A_{4} K_{1}^{11 / 10} K_{3} d_{1}^{11 / 10} \gamma^{1 / 2}+A_{5} K_{1}^{11 / 30} K_{4} d_{1}^{-3 / 10} \gamma^{1 / 6},
\end{aligned}
$$

where $y=\int_{0}^{1}\left[\left(d_{1} P_{x}\right)^{2}+\left(d_{1} Q_{x}\right)^{2}\right] d x$. The inequality (2.21) implies that there exist $\tilde{\tau}_{2}>0$ and positive constant $\widetilde{M_{2}}$ depending on $d_{i}, \alpha_{i j}, a_{i}, b_{i j}, e_{i}(i, j=1,2)$, such that

$$
\int_{0}^{1}\left(d_{1} P_{x}\right)^{2} d x, \int_{0}^{1}\left(d_{1} Q_{x}\right)^{2} d x \leq \widetilde{M}_{2}, t \geq \tilde{\tau}_{2}
$$

In the case that $d_{1}, d_{2} \geq 1, \xi \in[\underline{d}, \bar{d}]$, the coefficients of inequality (2.20) can be estimated by some constants which depend on $\underline{d}, \bar{d}$, but do not depend on $d_{1}, d_{2}$. So $\widetilde{M_{2}}$ depends on $\alpha_{i j}, a_{i}, b_{i j}, e_{i}(i, j=1,2), \underline{d}$ and $\bar{d}$, but it is irrelevant to $d_{1}, d_{2}$, when $d_{1}, d_{2}$ $\geq 1$ and $\xi \in[\underline{d}, \bar{d}]$. Since

$$
\left(\begin{array}{c}
P_{x} \\
Q_{x}
\end{array}\right)=\left(\begin{array}{cc}
P_{u} & P_{v} \\
Q_{u} & Q_{v}
\end{array}\right)\left(\begin{array}{c}
u_{x} \\
v_{x}
\end{array}\right)
$$

we can transform the formulations of $u_{x}, v_{x}$ into fraction representations, then distribute the denominators of the absolute value of the fractions to the numerators item and enlarge the term concerning with $u_{x}, v_{x}$ to obtain 


$$
\left|d_{1} u_{x}\right|+\left|d_{1} v_{x}\right| \leq L\left(\left|d_{1} P_{x}\right|+\left|d_{1} Q_{x}\right|\right), \quad 0<x<1, t>0,
$$

where $L$ is a constant depending only on $\xi, \alpha_{i j}(i, j=1,2)$. After scaling back and contacting estimates (2.22) and (2.23), there exist positive constant $M_{2}$ depending on $d_{i}, \alpha_{i j}, a_{i}, b_{i j}, e_{i}(i, j=1,2)$ and $\tau_{2}>0$, such that

$$
\int_{0}^{1} u_{x}^{2} d x, \int_{0}^{1} v_{x}^{2} d x \leq M_{2}, \quad t \geq \tau_{2}
$$

When $d_{1}, d_{2} \geq 1$ and $\xi \in[\underline{d}, \bar{d}], M_{2}$ is independent of $d_{1}, d_{2}$.

(ii) $t \geq 0$. Modifying the dependency of the coefficients in inequalities (2.12)-(2.14), namely replacing $M_{0}, M_{1}$ with $M_{0}^{\prime}, M_{1}^{\prime}$, there exists a positive constant $M_{2}^{\prime}$ depending on $d_{i}, \alpha_{i j}, a_{i}, b_{i j}, e_{i}(i, j=1,2)$ and the $W_{2}^{1}$-norm of $u_{0}, v_{0}$, such that

$$
\int_{0}^{1} u_{x}^{2} d x, \int_{0}^{1} v_{x}^{2} d x \leq M_{2}^{\prime}, \quad t \geq 0
$$

Furthermore, in the case that $d_{1}, d_{2} \geq 1, \xi \in[\underline{d}, \bar{d}], M_{2}^{\prime}$, depends on $\underline{d}, \bar{d}$, but does not depend on $d_{1}, d_{2}$.

Summarizing estimates (2.5), (2.9), (2.24), and Sobolev embedding theorem, there exist positive constants $M, M$ ' depending only on $d_{i}, \alpha_{i j}, a_{i}, b_{i j}, e_{i}(i, j=1,2)$, such that (1.10) and (1.11) hold. In particular, $M, M^{\prime}$ depend only on $\alpha_{i j}, a_{i}, b_{i j}, e_{i}(i, j=1,2), \underline{d}$ and $\bar{d}$, but do not depend on $d_{1}, d_{2}$, when $d_{1}, d_{2} \geq 1$ and $\xi \in[\underline{d}, \bar{d}]$. Similarly, according to (2.5)', (2.9)', (2.24)', there exists a positive constant $M$ "' depending on $d_{i}, \alpha_{i j}, a_{i}$, $b_{i j}, e_{i}(i, j=1,2)$ and the initial functions $u_{0}, v_{0}$, such that

$$
|u(\cdot, t)|_{1,2,},\left.v(\cdot, t)\right|_{1,2} \leq M^{\prime \prime}, \quad t \geq 0 .
$$

Further, in the case that $d_{1}, d_{2} \geq 1, \xi \in[\underline{d}, \bar{d}], M^{\text {u }}$ depends only on $\underline{d}, \bar{d}$, but do not depend on $d_{1}, d_{2}$. Thus, $T=+\infty$.

This completes the proof of Theorem 1.1.

\section{Global stability}

In order to obtain the uniform convergence of the solution to system (1.2), we recall the following result which can be found in [21].

Lemma 3.1. Let $a$ and $b$ be positive constants. Assume that $\phi, \psi \in C^{1}([a,+\infty)), \psi(t)$ $\geq 0$ and $\phi$ is bounded from below. If $\phi^{\prime}(t) \leq-b \psi(t)$ and $\psi^{\prime}(t)$ is bounded from above in $[a,+\infty)$, then $\lim _{t \rightarrow \infty} \psi(t)=0$.

Proof of Theorem 1.2. Let $(u, v)$ be a solution for the system (1.8) with initial functions $u_{0}(x), v_{0}(x) \geq(\mathbb{\nabla}) 0$. From the strong maximum principle for parabolic equations, it is not hard to verify that $u, v>0$ for $t>0$. Define the function

$$
H(u, v)=\int_{0}^{1}\left(u-u^{*}-u^{*} \ln \frac{u}{u^{*}}\right) d x+\int_{0}^{1}\left(v-v^{*}-v^{*} \ln \frac{v}{v^{*}}\right) d x .
$$


Then the time derivative of $H(u, v)$ for the system (1.8) satisfies

$$
\begin{aligned}
\frac{d H}{d t}= & \int_{0}^{1} \frac{u-u^{*}}{u} u_{t} d x+\int_{0}^{1} \frac{v-v^{*}}{v} v_{t} d x \\
= & -\int_{0}^{1}\left[\frac{u^{*}}{u^{2}}\left(d_{1}+2 \alpha_{11} u+\alpha_{12} v\right) u_{x}^{2}+\left(\frac{\alpha_{12} u^{*}}{u}+\frac{\alpha_{21} v^{*}}{v}\right) u_{x} v_{x}+\frac{\alpha v^{*}}{v^{2}}\left(d_{2}+\alpha_{21} u+2 \alpha_{22} v\right) v_{x}^{2}\right] d x \\
& -\int_{0}^{1}\left[\left(u-u^{*}\right)^{2}\left(b_{11}+e_{1} v\right)+\left(b_{12}+b_{21}+e_{1} u^{*}+e_{2} v^{*}\right)\left(u-u^{*}\right)\left(v-v^{*}\right)+\left(v-v^{*}\right)^{2}\left(b_{22}+e_{2} u\right)\right] d x .
\end{aligned}
$$

The first integrand in the right-hand side of (3.1) is positive definite if

$$
4 u^{*} v^{*}\left(d_{1}+2 \alpha_{11} u+\alpha_{12} v\right)\left(d_{2}+\alpha_{21} u+2 \alpha_{22} v\right)>\left(\alpha_{12} u^{*} v+\alpha_{21} v^{*} u\right)^{2}
$$

and the condition (1.12) implies (3.2). The second integrand in the right-hand side of (3.1) is positive definite if

$$
4\left(b_{11}+e_{1} v\right)\left(b_{22}+e_{2} u\right)>\left(b_{12}+b_{21}+e_{1} u^{*}+e_{2} v^{*}\right)^{2}
$$

and the condition (1.13) implies (3.3). Consequently, there exists $\delta>0$, such that

$$
\frac{d H}{d t} \leq-\delta \int_{0}^{1}\left[\left(u-u^{*}\right)^{2}+\left(v-v^{*}\right)^{2}\right] d x, \quad \frac{d H}{d t} \leq 0, \quad(u, v) \neq\left(u^{*}, v^{*}\right) .
$$

By the maximum-norm estimate in Theorem 1.1 and some tedious calculations, we can verify $(d / d t) \int_{0}^{1}\left[\left(u-u^{*}\right)^{2}+\left(v-v^{*}\right)^{2}\right] d x$ is bounded from above. Then from Lemmas 3.1 and (3.4), we obtain

$$
\lim _{t \rightarrow \infty} \int_{0}^{1}\left(u-u^{*}\right)^{2} d x=\lim _{t \rightarrow \infty} \int_{0}^{1}\left(v-v^{*}\right)^{2} d x=0 .
$$

It follows from (3.5) and Gagliardo-Nirenberg-type inequality $|u|_{\infty} \leq C|u|_{1,2}^{1 / 2}|u|_{2}^{1 / 2}$ that $(u, v)$ converges uniformly to $\left(u^{*}, v^{*}\right)$. By the fact that $H(u, v)$ is decreasing for $t \geq$ 0 , it is obvious that $\left(u^{*}, v^{*}\right)$ is globally asymptotically stable. So the proof of Theorem 1.2 is completed.

\section{Acknowledgements}

This study was supported by the China National Natural Science Foundation (No. 11161041), the Fundamental Research Funds for the Central Universities (No. zyz2012074). The author also wishes to thank the referee for some valuable comments and helpful suggestions.

\section{Competing interests}

The author declares that they have no competing interests.

\section{Received: 3 May 2012 Accepted: 27 June 2012 Published: 27 June 2012}

\section{References}

1. Chattopadhyay, J: Effect of toxic substances on a two-species competitive system. Ecol Model. 84, $287-289$ (1996). doi:10.1016/0304-3800(94)00134-0

2. Tian, C, Zhang, L, Lin, Z: Pattern formation for a model of plankton allelopathy with cross-diffusion. J Franklin Instit. 348 , 1947-1964 (2011). doi:10.1016/j.jfranklin.2011.05.013

3. Kuto, K: Stability of steady-state solutions to a prey-predator system with cross-diffusion. J Diff Equ. 197, 293-314 (2004). doi:10.1016/j.jde.2003.10.016

4. Pang, PYH, Wang, M: Strategy and stationary pattern in a three-species predator-prey model. J Diff Equ. 200, 245-273 (2004). doi:10.1016/j.jde.2004.01.004 
5. Shi, J, Xie, Z, Little, K: Cross-diffusion induced instability and stability in reaction-diffusion systems. J Appl Anal Comput. 1, 95-119 (2011)

6. Choi, YS, Lui, R, Yamada, Y: Existence of global solutions for the Shigesada-Kawasaki-Teramoto model with weak crossdiffusion. Discrete Contin Dyn Syst. 9, 1193-1200 (2003)

7. Choi, YS, Lui, R, Yamada, Y: Existence of globle solutions for the Shigesada-Kawasaki-Teramoto model with strongly coupled cross-diffusion. Discrete Contin Dyn Syst. 10, 719-730 (2004)

8. Fu, S, Wen, Z, Cui, S: Uniform boundedness and stability of global solutions in a strongly coupled three-species cooperating model. Nonlinear Anal RWA. 55, 1-18 (2006)

9. Le, D: Cross-diffusion systems on $n$ spatial dimensional domains. J Indiana Univ Math. 51, 625-643 (2002)

10. Le, D, Nguyen, L, Nguyen, T: Shigesada-Kawasaki-Teramoto model on higher dimensional domains. Electron J Diff Equ. 72, 1-12 (2003)

11. Lou, Y, Ni, W, Wu, Y: On the global existence of a cross-diffusion system. Discrete Contin Dyn Syst. 4, 193-203 (1998)

12. Tuoc, PV: Global existence of solutions to Shigesada-Kawasaki-Teramoto cross-diffusion systems on domains of arbitrary dimensions. Proc Am Math Soc. 135, 3933-3941 (2007). doi:10.1090/50002-9939-07-08978-2

13. Yamada, Y: Existence of global solutions for the Shigesada-Kawasaki-Teramoto model with cross-diffusion. Math Anal Instit Commun Waseda Univ. 1358, 24-33 (2004)

14. Barrett, JW, Blowey, JF: Finite element approximation of a nonlinear cross-diffusion population model. Numer Math. 98, 195-221 (2004)

15. Chen, L, Jungel, A: Analysis of a parabolic cross-diffusion population model without self-diffusion. J Diff Equ. 224, 39-59 (2004)

16. Galiano, G, Garzon, ML, Jungel, A: Semi-discretization and numerical convergence of a nonlinear cross-diffusion population model. Numer Math. 93, 655-673 (2003). doi:10.1007/s002110200406

17. Gilardi, G, Stefanelli, U: Time-discretization and global solution for a doubly nonlinear Volterra equation. J Diff Equ. 228, 707-736 (2006). doi:10.1016/j.jde.2005.12.010

18. Amann, H: Dynamic theory of quasilinear parabolic equations-I. Abstract evolution equations. Nonlinear Anal. 12, 895-919 (1988). doi:10.1016/0362-546X(88)90073-9

19. Amann, H: Dynamic theory of quasilinear parabolic equations-II. Reaction-diffusion. Diff Integral Equ. 3, 13-75 (1990)

20. Amann, H: Dynamic theory of quasilinear parabolic equations-III. Global existence. Math Z. 202, 219-250 (1989). doi:10.1007/BF01215256

21. Wang, M: Nonlinear Partial Differential Equations of Parabolic Type. Science Press, Beijing (1993) in Chinese

22. Shim, S: Uniform boundedness and convergence of solutions to cross-diffusion systems. J Diff Equ. 185, 281-305 (2002). doi:10.1006/jdeq.2002.4169

23. Shim, S: Uniform boundedness and convergence of solutions to the systems with cross-diffusion dominated by selfdiffusion. Nonlinear Anal RWA. 4, 65-86 (2003). doi:10.1016/S1468-1218(02)00014-7

24. Shim, S: Uniform boundedness and convergence of solutions to the systems with a single nonzero cross-diffusion. J Math Anal Appl. 279, 1-21 (2003). doi:10.1016/50022-247X(03)00045-3

25. Nirenberg, L: On elliptic partial equations. Ann Scuola Norm Sup Pisa. 13, 115-162 (1959)

doi:10.1186/1687-1847-2012-89

Cite this article as: Jiao: Global behavior for a strongly coupled model of plankton allelopathy. Advances in Difference Equations 2012 2012:89.

\section{Submit your manuscript to a SpringerOpen ${ }^{\circ}$ journal and benefit from:}

Convenient online submission

- Rigorous peer review

- Immediate publication on acceptance

- Open access: articles freely available online

- High visibility within the field

- Retaining the copyright to your article

Submit your next manuscript at $\boldsymbol{\wedge}$ springeropen.com 